\title{
Habitability of Small Bodies - State of Knowledge and Motivations for Exploration in the Next Decade
}

Community White Paper for the Planetary Decadal Survey, 2023-2032

\author{
A White Paper commissioned \\ by the Small Bodies Assessment Group (SBAG)
}

Lead Author: Julie Castillo-Rogez (Jet Propulsion Laboratory, California Institute of Technology) Julie.C.Castillo@jpl.nasa.gov

Contributors: Jason D. Hofgartner (JPL/Caltech), Kelsi Singer (SwRI), Charles Cockell (U. of Edinburgh, UK), Bryan J. Holler (STSCI), Marc Neveu (GSFC, U. Maryland), Maitrayee Bose (ASU), Tim Swindle (U. Arizona, Tucson), Carly Howett (SwRI), Joseph Lazio (JPL/Caltech), John Elliott (JPL/Caltech), Jennifer Scully (JPL/Caltech), Andreas Nathues (MPS)

Co-Signers: Terik Daly (JHU/APL), David A. Williams (ASU), Simon Friesen (U. Waterloo, Canada), Jian-Yang Li (PSI), Lynnae C. Quick (NASA Goddard Space Flight Center), Katrin Krohn (German Aerospace Center Berlin - DLR), Mauro Ciarniello (INAF-IAPS, Rome, Italy), Alicia Neesemann (Freie Universität Berlin), Andrea Raponi (INAF-IAPS, Rome, Italy), Kynan Hughson (Georgia Institute of Technology), Debra Buczkowski (Johns Hopkins Applied Physics Laboratory), Rutu Parekh (German Aerospace Center Berlin - DLR), Michelangelo Formisano (INAF-IAPS, Rome, Italy)

Acknowledgements: A portion of this research was carried out at the Jet Propulsion Laboratory, California Institute of Technology, under a contract with the National Aeronautics and Space Administration. 


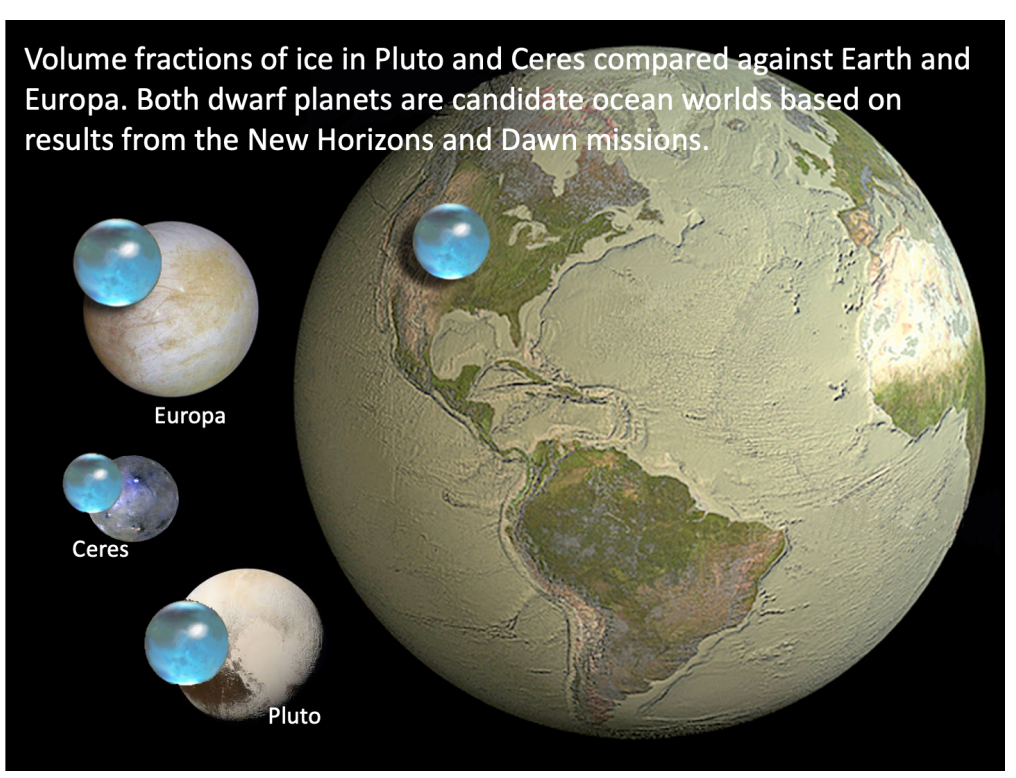

Executive Summary

This white paper addresses the following priority question identified by NASA's Small Bodies Assessment Group: Do sustainable habitable environments exist on any of the small bodies? This question is motivated by the results of the Dawn and New Horizons missions at Ceres and the Pluto system during the past decade. These missions revealed a class of objects that could potentially be habitable in the past and at present given the occurrence of internal oceans. "Sustainability" is key here as these bodies are presumably heat-starved in the absence of recent tidal heating. The mechanisms that contributed to the preservation of liquid inside dwarf planets and their recent, and potentially ongoing activity, remain to be fully comprehended. Future investigations should confirm conditions for present-day habitability (e.g., liquid abundance and environmental conditions) and identify mechanisms that drive endogenic activity. This is particularly critical as abundant organic matter has been found at these objects. It is likely that many other bodies beyond Pluto's orbit are water- and organic-rich and geologically active.

In order to pursue the exploration of small bodies of astrobiological value, recommendations for the next decade are (in no priority order):

* To dedicate telescope time (including Vera C. Rubin Observatory and JWST) for the detailed surface characterization of dwarf planets and other large TNOs,

* To pursue theoretical, experimental, and analog research addressing small body habitability,

* To deploy a sample return mission to dwarf planet Ceres under the New Frontiers program,

* To deploy a Pluto Orbiter as a part of the Flagship program,

* To deploy a flyby mission to a dwarf planet or a large TNO in the Kuiper belt (possibly within the New Frontiers program and/or combined with other missions).

Specific technology developments and investments required to achieve these endeavors are addressed in separate white papers but include in particular:

* Experimental research on organics, brines, and super/hypervolatiles

* Development of instrumentation suitable for astrobiology affordable under the resource allocations of competitive mission solicitations

* Spacecraft technologies (consistent with OPAG's Recommendations)

- Maturation of radioisotope electric propulsion and development of fission reactors

- Continuation/augmentation of radioisotope production for radioisotope power systems

- Investment in telecommunication technologies

- Maturation of on-board data processing and autonomous operation technologies

We also recommend that the decadal survey consider the critical role of team dynamics, equity, diversity, inclusion, and accessibility in planetary science. 


\section{DISCOVERY OF PRESENTLY HABITABLE SMALL BODIES: A MAJOR OUTCOME FROM THE TWO PREVIOUS DECADAL SURVEYS}

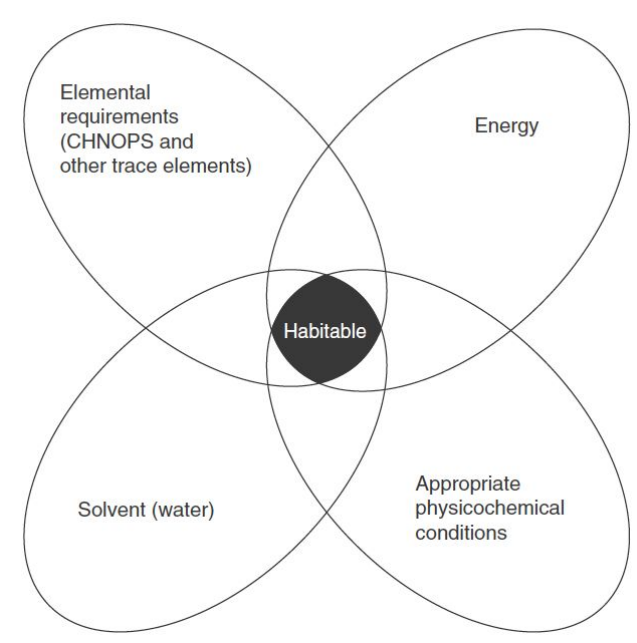

Habitability refers to the ability of an environment to support life (which must include reproduction if a population of organisms is to be sustained). The habitability of any small body is determined by the presence of key ingredients that are defined by life as we know it (Figure 1): liquid water as a solvent, basic elements needed to construct macromolecules (CHNOPS elements and other elements that are organism-dependent) relevant for life or its sustenance, a source of energy and a set of physical and chemical conditions that lie within the currently known limits for life (see [1].)

Figure 1. Parameters defining planetary habitability [1].

Summary of Findings in the Last Decade

Recent findings have revealed the astrobiological significance of small bodies:

- Most water-rich small bodies hosted conditions amenable to prebiotic chemistry for some time in their history. Analyses of carbonaceous chondrites indicate organic matter evolution in hydrothermal environments $[2,3]$.

- Space missions to Ceres and Pluto and ground-based observations of dwarf planets (DP) indicate that the occurrence of liquid water might be a universal feature of DPs:

- Geological and chemistry observations suggest that Ceres hosts brines below its crust [e.g., 4] and Pluto could hold a deep liquid reservoir at present [5].

- That class of objects have adequate long-lived radionuclides that maximize the longevity of habitable conditions (radioisotope decay heat) but require low-eutectic compounds and hydrates to help extend the lifetime of oceans and/or regional seas [6].

- The characteristics of Haumea also suggest it has been hosting a deep ocean [7].

- A rich chemistry and a large abundance of organic compounds have been found at Ceres [8], Pluto [e.g., 9], and is suspected at other DPs and large TNOs.

- Ceres and Pluto both show recent and ongoing geological activity $[8,10,11]$ and other DPs and large TNOs are hypothesized to be active [12].

- Organic matter has been recently recognized as an important component of the mantles of large icy moons and DPs [13].

\section{Significance}

The Dawn and New Horizons missions expanded the realm of ocean worlds beyond the Earth and the icy moons of the giant planets. Ceres is categorized as an "ocean world" and Pluto as a "candidate ocean world" in the Roadmap to Ocean Worlds (ROW, [14]). Ceres is the most water-rich object in the inner solar system after Earth, and its astrobiological exploration is achievable within the resources of a New Frontiers program [15]. Pluto is the largest and best-studied representative of DPs from the Kuiper Belt. Both bodies are small enough that their 
oceans are in direct contact with their rocky mantles. Solar dynamical models and astronomical observations strongly drive the idea that volatiles and organics were brought to the inner solar system by outer solar system planetesimals and protoplanets, of which Ceres and Pluto may be surviving representatives [16]. [17] also recently suggested that Earth got the majority of its volatiles from a Ceres-sized body.

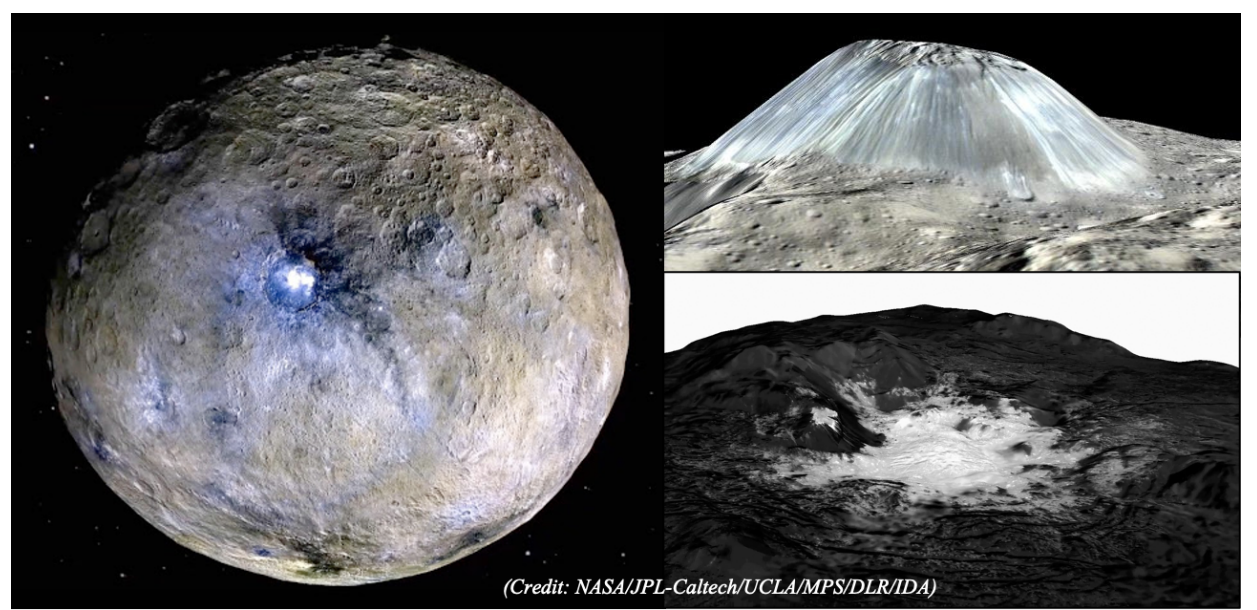

Figure 2. Key attributes of Ceres reveal its habitability potential.

Left: Enhanced color imaging highlights regions rich in salts, focusing on the recently emplaced Occator faculae.

Top Right: Ahuna Mons (<100 Mya) is a $4-\mathrm{km}$ high, $17-\mathrm{km}$ wide mountain that displays sodium carbonates. Gravity data suggest a source from the mantle and is best explained by the upwelling of low-viscosity material [10]. Bottom Right: Cerealia Facula (<20 Mya) is a 12 km broad region enriched in sodium carbonate, ammonium chloride, and hydrohalite [8]. The latter is not stable on Ceres' surface and had to be emplaced recently. The mechanisms driving material exposure at Ceres is not fully understood but requires access to a long-lived brine region [e.g., 18$]$.

\section{Key Questions Arising from Mission and Ground-based of DPs/Large TNO Observations}

- What are the characteristics (physical, compositional) of the liquid environments in Pluto (if any) and Ceres?

- Are there any other bodies out there, either in the main-belt or beyond that host(ed) global brine oceans or seas?

- What drives the long-term preservation of liquid in bodies without tidal heating?

- Are DP oceans habitable or do they exist because of concentrated brines and second-phase impurities that may not be hospitable to life?

- What is the extent of prebiotic chemistry in DPs/TNOs?

- What are the correlations between the composition and spectral properties of DPs/TNOs?

- What are the physical properties and key processes driving DP/TNO internal and surface properties and how are they modified over time?

- Is material transfer from the deep interior to the surface occurring in DP/TNOs?

- How does abundant organic matter impact the evolution of DPs and TNOs interiors?

- Did complex organic molecules produced via space weathering at the surface of Pluto and TNOs have the opportunity to access oceans in these bodies (e.g., via large impacts)? 


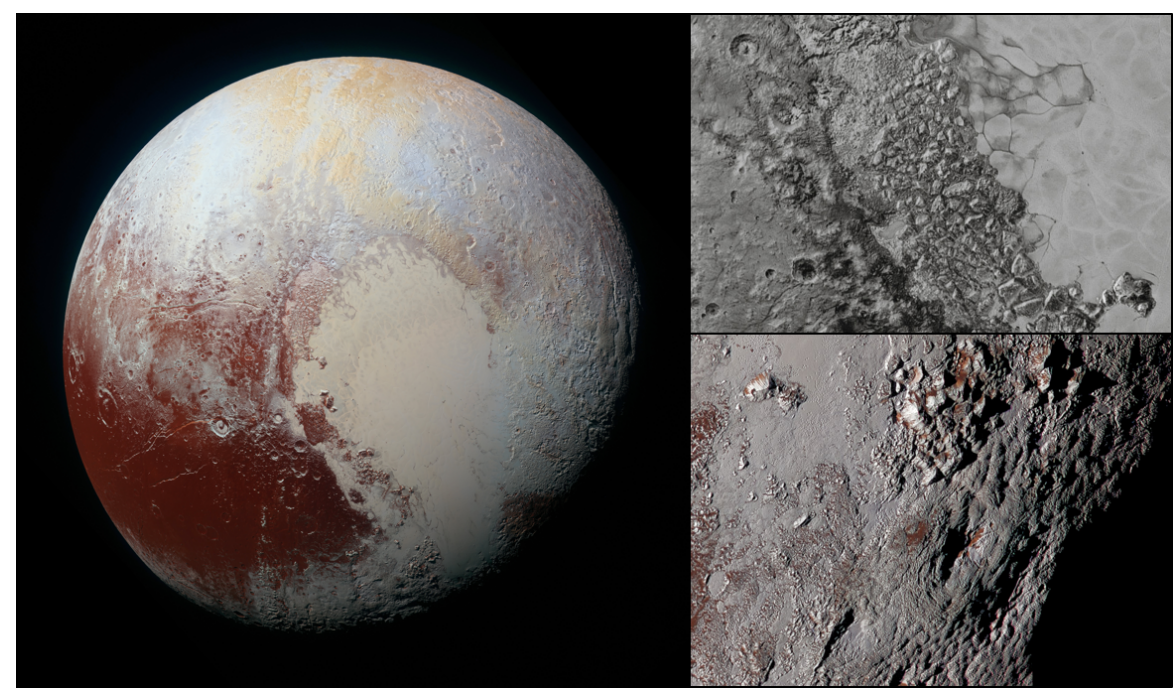

Figure 3. Pluto displays a wide range of terrain ages; some regions have few or no impact craters, implying relatively recent resurfacing and ages <30 Mya [19-21]. Resurfacing appears to occur through both exogenic and endogenic processes. One example is the very large annular massifs (one is shown at lower right) that may represent a unique form

of icy volcanism [20, 22]. This may require more internal heat than was originally expected for dwarf planets (especially later in their histories). Pluto also has a lot of carbon-rich materials across its surface, including $\mathrm{CO}, \mathrm{CH}_{4}$ and various tholins, or heavier hydrocarbons from the processing of $\mathrm{CH}_{4}$ (represented by the dark, red and yellow materials in the enhanced color image). Image credit: NASA/JHU APL/SwRI.

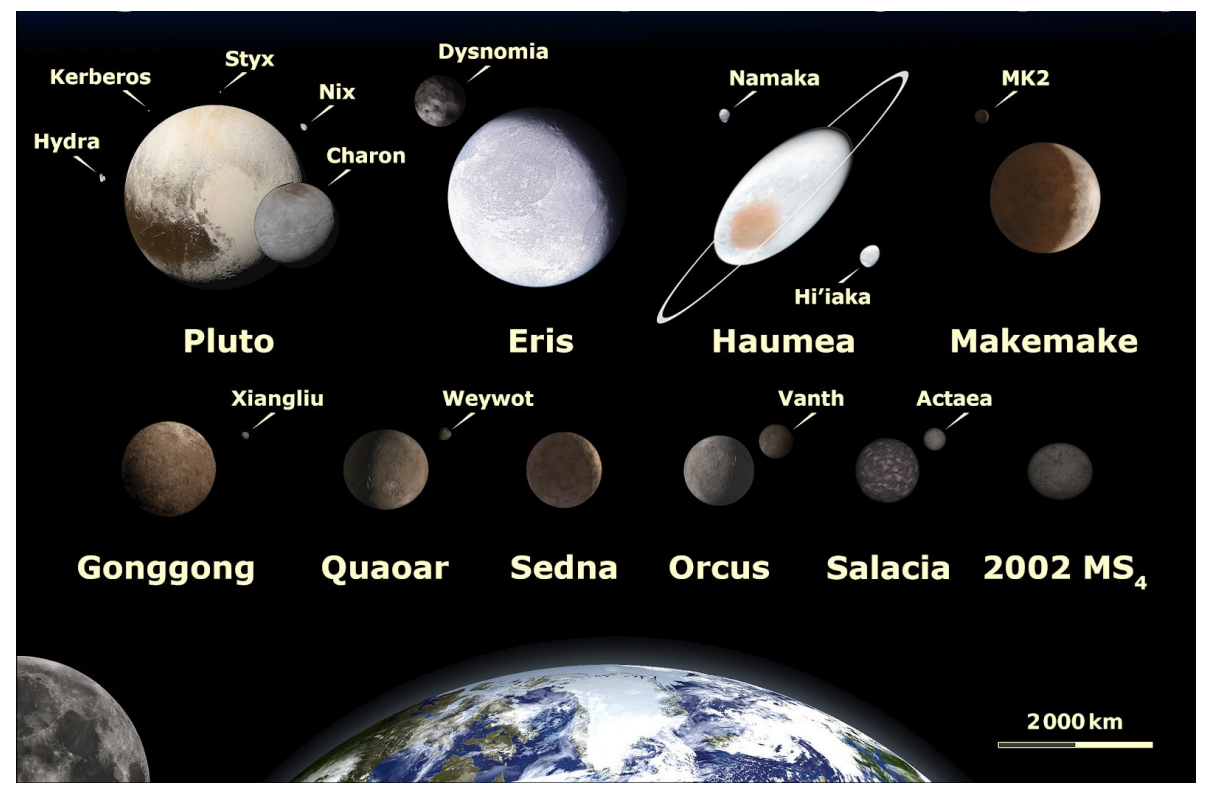

Figure 4: Largest detected TNOs These worlds have diverse heliocentric orbits, moon and ring systems, surface volatile abundances and atmospheres, surface textures, and likely different evolutionary histories.

Pluto is the largest and only system that has been explored during a flyby; an orbiter mission in the flagship program

recommended. A flyby of one or more of these other large TNOs, possibly in the New Frontiers program, and/or possibly combined with other mission architectures, is recommended. Some of these objects may harbor subsurface oceans and some have complex organics at their surface; determining their habitability should be included in future investigations of these worlds. Image credit: https://en.wikipedia.org/wiki/File:EightTNOs.png 


\section{PRIORITIES FOR THE ASTROBIOLOGICAL EXPLORATION OF DWARF PLANETS AND LARGE TNOS IN THE NEXT DECADE}

\section{- Progress along the Roadmap for Ocean Worlds for Ceres and Pluto:}

See white papers on Future Ceres Exploration [23] and Pluto Follow-on Missions [24]

$\circ$ Confirm the presence and scale of liquids inside Ceres and Pluto and identify the source of ongoing activity at these bodies. Subsurface oceans or regional brine pockets can be detected via geophysical techniques or analysis of geological features, from landed and orbital missions. Detailed morphology and gravity analysis can advance the understanding of activity drivers [23, 25]. Comparisons between Pluto and past ocean world Charon help assess what factors have maintained Pluto's potential habitability (e.g., size effect).

$\circ$ Characterize liquid environments via orbital, in situ, and/or (for the case of Ceres) sample return missions.

- Search for evidence of material transfer between interior and surface.

- Characterize complexity of organic matter, in contact with water (aqua-organo-reactions) or altered by radiation

- Pursue the characterization of dwarf planets in the Kuiper Belt, e.g., search for evidence of geologic activity and deep oceans and quantify volatile and organic makeup.

High-Priority Space Missions (NF = New Frontiers; FS = Flagship)

\begin{tabular}{|c|c|c|c|c|}
\hline Mission Target and Scope & Class & Priority Investigations & Required Technologies & Refs \\
\hline $\begin{array}{l}\text { Ceres sample return from } \\
\text { recent evaporite (incl. orbital } \\
\& \text { in situ characterization) }\end{array}$ & $\mathrm{NF}$ & \multirow{2}{*}{$\begin{array}{l}\text { Search for deep brines } \\
\text { Characterize the composition } \\
\text { of organic- and salt-rich } \\
\text { material recently exposed on } \\
\text { the surface; characterization } \\
\text { of past and ongoing activity } \\
\text { on global scale }\end{array}$} & \multirow{2}{*}{$\begin{array}{l}\text { Targeted landing, } \\
\text { Sampling system, } \\
\text { Back planetary } \\
\text { protection-compliant } \\
\text { sample handling } \\
\text { Cold temperature } \\
\text { curation }\end{array}$} & [14] \\
\hline $\begin{array}{l}\text { Ceres in situ exploration at } \\
\text { multiple sites (e.g., evaporites) }\end{array}$ & NF & & & {$[14]$} \\
\hline Pluto Orbiter & FS & $\begin{array}{l}\text { Interiors of Pluto and Charon; } \\
\text { Evolution of surfaces and } \\
\text { atmospheres in Pluto system }\end{array}$ & \multirow{2}{*}{$\begin{array}{l}\text { Next-Gen RTGs, } \\
\text { Nuclear fission } \\
\text { generator, high- } \\
\text { throughput EP thrusters } \\
\text { Next generation telecom } \\
\text { systems } \\
\text { Autonomous operations }\end{array}$} & {$[26]$} \\
\hline $\begin{array}{l}\text { Dwarf planet flyby (in Kuiper } \\
\text { Belt) }\end{array}$ & $\begin{array}{c}\text { NF or } \\
\text { with Pluto } \\
\text { FS }\end{array}$ & $\begin{array}{l}\text { Evolution of KBO population; } \\
\text { Extent of ongoing endogenic } \\
\text { vs. solar-driven activity }\end{array}$ & & {$[26]$} \\
\hline
\end{tabular}

\section{Enabling Technologies}

- Maturation and performance improvement of radioisotope electric propulsion (EP): Current MMRTGs are not practical. EP for demanding missions will be enabled by the development of the Next Generation RTG, nuclear fission generator, or advanced dynamic radio power systems and high-throughput EP thrusters. The development of Nuclear EP using a $10 \mathrm{~kW}$ kilopower-reactor power system would be enabling for whole new classes of missions at these distant ranges [27]. Continuation/augmentation of radioisotope production for RPS is critical to the future exploration of Pluto and the Kuiper Belt. 
- Improved telecommunication systems: (a) Ka band ( $\sim 32 \mathrm{GHz})$ provides both increased data bandwidths and value for radio or gravity science by reducing the effects of the interplanetary medium. (b) Expand the capability of Deep Space Optical Communications after demonstration on the Psyche mission: e.g., increase in the flight terminal aperture size (from $20 \mathrm{~cm}$ to $50 \mathrm{~cm}$, e.g., HiRISE camera), improvements in the optical power amplifier average output level (from $5 \mathrm{~W}$ to $50 \mathrm{~W}$ ) and in efficiency (from $15 \%$ to $30 \%$ ).

- Keeping the $70 \mathrm{~m}$ DSN dishes going and upgraded per the the DSN roadmap. By the middle of the 2020s, the DSN will have the equivalent of *two* $70 \mathrm{~m}$ apertures at each complex (170 $\mathrm{m}$ antenna $+4 \times 34 \mathrm{~m}$ antennas). This capability represents the minimum necessary to conduct the various planetary missions over at least the next decade; it enables missions to TNOs, particularly if antenna arraying is used.

- Maturation of on-board data processing and autonomous operation technologies to enable/improve science return from a mission at Pluto and in the Kuiper belt [28, 29].

Telescopic observations for investigating the habitability of DPs and TNOs: Ground- and space-based telescope facilities can make significant contributions to the characterization of DPs and TNOs for the purpose of evaluating targets for future in-situ exploration. This includes high spatial resolution imaging in the visible for surface feature detection, near-infrared spectroscopy for surface composition, mid-infrared thermal studies for size determination, and identification and characterization of satellite orbits for mass and density estimates of the primary.

- Access to Hubble Space Telescope, which will continue to be the standard-bearer for studies of DPs and TNO binaries throughout the remainder of its operating lifetime. The UVIS mode of the WFC3 imager has revolutionized the detection and characterization of TNO satellites and their orbits and led to mass and density estimates for many primaries, which are important quantities for constraining internal composition and structure.

- Access to James Webb Space Telescope (JWST): NIRSpec (2.5-5 micron) is critical to the study of DP and TNO surface compositions (e.g., detection of organic compounds, water ice abundance and phase, trace species such as ammonia hydrates and salts) that may be indicative of past or present subsurface reservoirs. MIRI (5-28 micron), on the short-wavelength side of the thermal peaks of many TNOs, can be used to constraint target size and albedo, similar to previous investigations using the Spitzer Space telescope. [30-33]

- Access to large ground-based facilities: Monitoring of compositional changes on DPs, TNOs, and their large satellites can be carried out over baselines of many years using a variety of facilities and instruments, including IRTF/SpeX, Gemini/GNIRS, and Keck/NIRES, to name a few Keck/OSIRIS and VLT/SINFONI imaging spectrometers, combined with adaptive optics systems. Satellite orbit characterization for binaries with wider separations and even surface mapping of DPs can be performed with Keck/NIRC2, Gemini/GMOS and NIRI and VLT/SPHERE. ALMA for surface/subsurface thermal conditions and composition, and outgassing search.

Required Supporting Activities (list not exhaustive).

Theoretical Research

- Understanding elemental cycles, in particular of carbon and nitrogen.

- Modeling of geologic and atmospheric processes on Ceres, Pluto, and other large TNOs. 
- Modeling of aqueous and geophysical processes and of energy availability. Experimental Research:

- Optical and dielectric constants of relevant volatiles and minerals, including organic compound for the interpretation of ground-based observations

- Mechanical and thermal properties of volatiles and organics

- Investigations at analog sites on Earth [see Ceres WP for specific recommendations]

- Geochemical and microbiological experimental studies of analog brine solutions to investigate habitability and limits of life in small-body aqueous environments.

\section{Data Analysis}

- Increase funding for data analysis programs to expand the science return of space missions, in general, and of the New Horizons and Dawn missions in particular.

- Continue support of the Solar System Observations (SSO) and Solar System Workings (SSW) ROSES programs for the analysis of ground- and space-based observations.

\section{Diverse community and internal collaborations}

- Plans to explore these and other important targets requires the involvement of a diverse, inclusive, and healthy community [32].

- The missions outlined above would benefit from international collaborations to increase science return and leverage expertise across the entire planetary science community.

REFERENCES (WP = White Papers) [1] Cockell+ (2016) Habitability: A review, Astrobiology 16:1. [2] Pizzarello+ (2015) The path of reduced nitrogen towards early Earth: The cosmic trail and its solar shortcuts, ApJ 814:107. [3] Vinogradoff+ (2018) Evolution of interstellar organic compounds under asteroidal hydrothermal conditions, Icarus 305, 358-370. [4] De Sanctis+ (2020) Recent emplacement of hydrated sodium chloride on Ceres from ascending salty fluids, Nat. Astron., Aug 10. [5] Nimmo+ (2016) Reorientation of Sputnik Planitia implies a subsurface ocean on Pluto, Nature 540, 94-96. [6] Castillo-Rogez (2020) Future exploration of Ceres as an ocean world, Nat. Astron., Aug 10. [7] Dunham+ (2019) Haumea's Shape, Composition, and Internal Structure, ApJ 877:41. [8] De Sanctis+ (2019) Characteristics of organic matter on Ceres from VIR/Dawn high spatial resolution spectra, MNRAS 482:2407-2421. [9] Cruikshank+ (2019) Prebiotic chemistry of Pluto, Astrobiology 19, 831-848. [10] Ruesch+ (2019) Slurry extrusion on Ceres from a convective mud-bearing mantle, Nat. Geosci. 12, 505-509. [11] Cruikshank+ (2019) Recent cryovolcanism in Virgil Fossae on Pluto, Icarus 330, 155-168. [12] Hofgartner+ (2020) Ongoing resurfacing of KBO Eris by volatile transport in local, collisional, sublimation atmosphere regime, Icarus 332, 52-61. [13] Neri+ (2020) A carbonaceous chondrite and cometary origin for icy moons of Jupiter and Saturn, EPSL 530, 115920. [14] Hendrix+ (2020) WP Ocean Worlds: A Roadmap for Science and Exploration. [15] Castillo-Rogez+ (2020) PMCS Report on the Exploration of Ceres' Habitability. [16] Raymond+ (2017) Origin of water in the inner Solar System: Planetesimals scattered inward during Jupiter and Saturn's rapid gas accretion, Icarus 297, 134-148. [17] Budde+ (2019) Molybdenum isotopic evidence for the late accretion of outer Solar System material to Earth, Nat. Astron. 3, 736-741. [18] Scully+ (2020) Formation of the bright faculae in Ceres' Occator crater via long-lived brine effusion in a hydrothermal system, Nat. Comm., Aug 10. [19] Moore+ (2016) The geology of Pluto and Charon through the eyes of New Horizons. Science 351, 1284-1293. [20] Robbins+ (2017) Craters of the Pluto-Charon system, Icarus 287, 187-206. [21] Singer+ (2019). Impact Craters on Pluto and Charon Indicate a Deficit of Small Kuiper Belt Objects, Science 363. [22] Singer+ (2020) Pluto and Charon Craters and Terrain Age Estimates. In: The Pluto System. University of Arizona Press, Tucson. [23] Castillo-Rogez+ (2020) WP Future Exploration of Ceres. [24] Robbins+ (2020) WP Pluto System Follow On Missions: Background, Rationale, and New Mission Recommendations. [25] Craft+ (2020) WP Cryovolcanism in the Solar System. [26] Howett+ (2020) Pluto Orbiter and Kuiper Belt Exploration Mission, PMCS Report. [27] Casani+ (2020) WP Enabling a New Generation of Outer Solar System Missions. [28] Amini+ (2020) WP Advancing the Scientific Frontier with Increasingly Autonomous Systems. [29] Theiling+ (2020) WP Non-Robotic Science Autonomy. [30] Milam+ (2016) JWST's plan for operations and instrument capabilities for observations in the solar system, PASP 128, 018001. [31] Métayer+ (2019) JWST/NIRSpec prospects on transneptunian objects. Front. Astron. Space Sci. 6, 8. [32] Milazzo+ (2020) WP DEIA 101: Why is diversity important? 